\title{
Complexity Analysis of Data Aggregation and Routing Algorithms for Automated Utility Management Using WSN
}

\author{
Raja Jitendra Nayaka, Rajashekhar Chanabasappa Biradar
}

School of Electronics and Communication, Reva University, Bangalore, India

Email address:

rjnayaka@gmail.com (R. J. Nayaka),dir@reveinstitution.org (R. C. Biradar)

To cite this article:

Raja Jitendra Nayaka, Rajashekhar Chanabasappa Biradar. Complexity Analysis of Data Aggregation and Routing Algorithms for Automated Utility Management Using WSN. International Journal of Sensors and Sensor Networks. Vol. 8, No. 1, 2020, pp. 11-22. doi: $10.11648 /$ j.ijssn.20200801.12

Received: May 18, 2020; Accepted: June 2, 2020; Published: June 17, 2020

\begin{abstract}
At present most of the houses in the country have the traditional electromechanical or digital utility usage meters, water meter, and gas meters. Presently most of the utility meter reading, billing system and utility management is not automated. The recent advances in the wireless sensor networks (WSNs) have made strong impact on the development of low cost remote monitoring systems. The WSN based remote automated utility management, remote meter reading and billing for future smart cities will enhance the quality and service by government. This increases the revenue of government. Due to unpleasant trend in the growth of congestion in urban areas, the smart utility meter data traffic aggregation and routing faces more challenges in the traditional automated service departments. In this work we propose an integrated architecture that include electricity, water and gas utility meters and discuss the methodology for Data Aggregation and Routing for Integrated Public Utility Services (IPUS-DAR) using WSN network. This work aims to integrate three types of utility meters and minimize redundant routing data in the network by applying data aggregation that improves traffic performance. We discuss the computational complexity of proposed Data Routing with Data Aggregation algorithm. The comparative analysis of proposed Data Routing with Data Aggregation methodology with previous methods is analyzed. We investigate performance metrics which include packet delivery ratio, end-to-end delay, jitter, throughput and energy consumption with respect to varying network size using QUALNET.
\end{abstract}

Keywords: Smart Metering Infrastructures (SMI), Electrical Gas Water Sensor Node (EWGSN), WSN, QoS, IPUS-DAR

\section{Introduction}

Presently, global metering service industry is heterogeneous one. There are multiple communication protocols and interface. Evolution of the electricity meters using microprocessor based technology has proprietary protocols, interfaces and frame formats are unique to the manufacturer. Another issue associated with difficulties in integration of different make of meters from various vendors in the legacy network. Frequent changes in the requirement of the utilities services over the period, the additional parameters and features have been added; this resulted in different versions of meters even from the same manufacturer. The users and service providers with these multiple versions of meters are burdened with multiple data formats on proprietary protocols [8]. At present, most of the houses across globe have the traditional electromechanical or digital watt hour meters, water meter and gas meter. These public utilities have individually managed by connected service departments. Presently, the billing system along with theft or leakage control and management of public utilities is not fully automated. Service provider handles individual meters reading manually at the end of each month, however finds difficult to trace theft and leakage of utilities. As number of meters grows, the manual collection of data and theft or leakage control becomes cumbersome task and time consuming and this leads to revenue loss [20]. In some places task become infeasible if the data terminals are unreachable. Therefore, a wireless sensor network based data collection mechanism is needed. The issues related with utility services task can be achieved by using wireless sensor communication network. WSN based AMR is a system and process used to remotely collect electrical, water and gas meter data without the physical presence of meter readers at the user premises. With such automation system it is possible to read multiple 
meters remotely at any time or predefined intervals and utility theft or leakage can be detected remotely. AMR is also known as smart meters and associated network is called smart grid [1-3]. It provides cost effective solution to meter reading services. In recent years, increased competition in the utilities service sectors has entailed changes in regulatory frameworks and structures of enterprises with value added services.

The creation of future smart cities involves enhancing the living quality and service performance of the city by government. Due to unpleasant trend in the growth of congestion in urban areas, smart utility meter data route planning becomes challenging for automated utility meter service departments. This is often accompanied with advancement in the development of mobile web services or phone apps to bring enhanced services to the people of a city in urban area.

Applications of WSN are wide; It is undergoing rapid technological changes. There is wide acceptance of Internet Protocol (IP) globally. WSNs will become the key technology for IoT. There is research scope to integrate WSN with the internet of things (IoT). By adopting IoT for data fusion for WSN, it is easier to process data and make meaningful use of information and closely couple with novel communication technologies [15-17]. As part of future work, we will evaluate the efficiency of network topology by introducing IoT (Internet of Things) at level-2 fusion center to provide QoS support in the larger network size. The Multihop WSN Networks have attracted significant academic interest in the last decade. The research in this area has sparked the development and commercialization of automated metered utility service solutions. This has resulted in technology advancement smart city automated utility management market. The combination of WSN and IoT solution will drive automated metering services [19]. Presently, The smart city planning that incorporates real time information gained from smart city sensors sent over the network. This is used for estimating future sensor traffic situations for effective route calculation. Typically, automated public utility network contains thousands of metered sensor data that have the ability to remotely transfer data to central BS. While automated utility network have limited by processing power and bandwidth, net-working a large number of such nodes gives rise to requirement of a reliable, high throughput, robust networking for accurate data transfer covering wider utility network.

The large amount of metered sensor data is transferred on automated utility network from each house to central station BS in multi-hop fashion. Efficient data routing in such network is challenging task due to large number of data are transmitted to BS from multiple metered sensor node. In this scenario, there is high probability of redundant data to be transmitted in the network. This will consume unnecessary network band width and energy. This data redundancy to be exploited by data aggregation along with routing protocol. The routing protocol should optimally exploit scarce resources such as limited bandwidth and energy, computing capability, packet processing delay, etc. in order to achieve efficient packet routing in the network $[1,2,8,9]$.

This work proposes Routing Protocol with Data aggregation for Integrated Public Utility Data Services using WSN (IPUS-DAR) to meet above challenges for varying automated utility network size. The proposed method of data aggregation techniques for data routing will reduce the control-data overhead for route discovery to enhance the throughput of the network.

The reminder of this work is organized as follows. In the section 2 Study of related works are discussed. Section 3 and 4 deals with the proposed methodology of data aggregation for routing protocol in integrated public utility data services using WSN. In the section 5 computational complexity of proposed Data Routing with Data Aggregation algorithm is discussed. In the section 6 Comparative performance analysis of proposed Data Routing with Data Aggregation Methodology with previous methods is analyzed along with results. We conclude with possible future research directions in section 7 .

\section{Related Works}

Due to popularity of wireless sensor network application, the extensive research and development of wireless data aggregation techniques and routing protocols are proposed to provide data communication in WSN based remote monitoring solutions. These are simulated in a environment using open source simulators for performance and comparative analysis of routing protocols. Many researchers have conducted performance analysis of individual meter data aggregation techniques or routing protocol that are carried out in GSM, W-MAX, ZigBee and WSN networks. For this reason, evaluating the performance of routing protocols with data aggregation algorithms in the simulation environment for utility meter data is still an active research area. In this section we study and analyze the related works proposed by researchers to find the gaps.

The author propose an ECWR (Energy and Congestion Aware Routing Metric for Smart Grid AMI Networks in Smart City) energy and congestion aware routing metric (ECRM) for Gas and Water smart meter networks. The proposed metric is an adaptive parent node selection mechanism that considers the residual energy and queue utilization of neighbouring nodes. In this work, the authors consider residual energy and queue utilization off neighbouring nodes to avoid routing loops and inconsistencies. Secondly, to avoid network congestion the queue utilization of the neighbouring nodes with minimum hop counts and unreliable links between the node and the root were also examined [3].

The proposed work on Hybrid Wireless Mesh Protocol (HWMP) is the default routing protocol used specifically for Wireless Mesh network consist of fixed nodes. HWMP is hybrid routing protocol supports reactive routing protocol and proactive routing protocol. The tree-based proactive routing protocol is adopted in network by using RMAODV 
AODV. The authors proposed Energy saving-based Hybrid Wireless Mesh Protocol (EHWMP) routing protocol by adjust the original HWMP protocol to meet the AMR requirements. This modification includes energy parameters of nodes, data packet header and original routing metric of HWMP [5].

The scholars proposed ORNP WSN SBMC (Optimal relay node placement in wireless sensor network for smart buildings metering and control). This mechanism examines WSN communication infrastructure for smart grid implementation in a building. A scheme has been proposed for the WSN deployment in buildings, in which sensors are massively placed to meter electricity consumption and collect illumination, thermal, pressure information, and multiple base stations are connected with the communication network for power grid distribution network. This scheme identifies the specific requirements of metering and control in buildings and provides optimal deployment of WSN. It exploits the simulation tool to simulate building environment and to test optimal deployed WSN [11]. Integer programming approaches for both deterministic and robust cases are considered. Researchers proposed CO WSNBI (Cost optimization of wireless enabled metering infrastructures). Advanced Metering Infrastructure (AMI) which measures, collects and analyzes information by communicating with metering devices such as electric meters, gas meters, temperature meters, and water meters, either on request or on a schedule. The Advanced Metering Infrastructure consists of a collection of neighbourhood Area Networks (NANs) that include smart, wireless-enabled mesh-connected meters or sensor nodes. Each NAN is controlled by a gateway or Access Point (AP). These devices, in turn, are usually meshconnected using wireless or wire line back-haul links to servers. This contribution develops an elegant graphtheoretic approach for optimizing the cost of an AMI by maximizing the ratio of the number of sensors nodes in a NAN to that of gateways or APs. A MATLAB program has been implemented to automate their approach which can deal with random and complex NAN topologies.

The AMR WMN SOPC (Automatic Meter Reading System Based on Wireless Mesh Networks and SOPC Technology AMR system used for automatic reading of the measure meters of energy consumption. A remote wireless AMR system based on wireless mesh networks and SOPC technology is presented in this mechanism to solve the problems in existing AMR system. The system consists of meters, wireless sensor nodes, data collector, management center and wireless communication networks. In this method, the data information is transmitted from the sensor nodes to the data collector using ZigBee communication. In this architecture, the system uses Ethernet transmit data from the data collector to the management center. The data collector acts as a gateway. This method adopts wireless mesh network topology. The systems presented in this mechanism has much significant excellence, such as networked, wireless, moveable and lower power consumption. The proposed system has a real application field for remote measure and management of Electric power, water supply, and gas supply. [12].

Authors have presented ICMBD WDN (An IoT System for Continuous Monitoring and Burst Detection in Intermittent Water Distribution Networks for continuous monitoring of burst and leakage in intermittent water distribution network) using WSN and IoT. In this system, the processed data are wirelessly transmitted to a central web server using the MQTT IoT protocol. The authors proposed adaptive Kalman filtering burst and leakage detection algorithm along with system node and server prototypes to study the performance [13].

Author investigated TEGPLC AMIN (Topology Effect on a G3-PLC based AMI Network) the effect of network topology on the performance of a PLC-based AMI system using the OMNeT network simulator [14]. Two different topologies such as centralized and distributed for an AMI network shows that topology has an impact on the performance of the system in based on decreasing the collision percentage on the DC switch.

The authors discussed APWCI (Application Protocols and Wireless Communication for IoT: A Simulation Case Study Proposal. The main application requirements for IoT such as Quality of Service (QoS) security mechanisms, discovery service resources and web integration [15]. They analyze the applicable protocols used such as DDS, MQTT-SN, XMPP, CoAP, and AMQP. The author analyzed the behavior of IoT applications Low Power Wide Area (LPWA) networks for smart meters, smart buildings, and smart cities.

Author proposed SMDI ZBWSN SG (A Smart Meter Design and Implementation Using ZigBee Based Wireless Sensor Network in Smart Grid). The Wireless Sensor Home Area Network (WSHAN) with WSN based ZigBee interfaced smart meter is designed and implemented. The proposed system measures energy usage real data logs [16].

The author designed IBSEM EEUSG (IOT Based Smart Energy Meter for Efficient Energy Utilization in Smart Grid) ESP 8266 12E, smart energy wireless meter reading system that can monitor and analyze the data periodically for efficient meter data communication over IoT. The metered data is uploaded periodically over IP cloud space called "Thinksspeak" and monitoring is done by customer and supplier [17].

Author proposed EADT PCWSN (An Efficient Approach for Data Transmission in Power-Constrained Wireless Sensor Network) optimization of radio data transmission of water metering over WSN to evaluate its impact on power saving. The data redundancy removal mechanism is applied on the metered data collected from 20,000 nodes [18].

Performance results of this research show that by substantial data transmission reduction can be achieved when compared to the current approaches. The non-transmission of redundant data in this approach is major power saving to increase in the lifespan of the battery can be accomplished.

Authors developed an ARTZ MWBADSC (Design and Implementation of an IoT Assisted Real-Time ZigBee Mesh WSN Based AMR System for Deployment in Smart Cities) 
IoT assisted real-time AMR system for deployment in smart cities. In this system, the end user nodes are electricity meters are supported by a ZigBee mesh WSN [19]. The basic node collects metered data periodically and transmit to gateway. The gateway transforms a data package from the ZigBee protocol to the TCP/IP protocol before transmitting it to the utility cloud. The data analytic for utility usage analysis.

Based on survey of above related work, The main objective of this work is to design and analyze aggregated data routing methodology required for integrated public utility services using WSN. Automated utility service all over the world is undergoing a significant transition in their grid from transmission of metered data to central server for data storage. The Public utility supply, metering, and automated distribution systems are constantly evolving. The smart cities present a big challenge for utility service departments to be responsible for reliable metered data routing and transmission. The data management and routing is among the most challenging task and power consuming operation that must be addressed [1, 2, 8, 9].

A Data Routing and Aggregation protocol for Integrated Public Utility Services IPUS-DAR using WSN is proposed, which can decrease the control overhead for route discovery and improve the throughput of the network. The proposed IPUS-DAR routing protocol is designed to improve the lifetime, latency and reliability through the usage of novel frame format from the source node to the sink. The IPUS-DAR routing scheme using WSN is proposed, where the frame format and routing technique reduces the data traffic in the network, and multipath technique provides the reliable path.

\section{Proposed Methodology}

In this section, we focus on the routing of aggregated data in the integrated public metered utility services that carries information of electricity, water and gas utilities usage data to improve the performance of smart utility grid. This method of routing protocol focuses on minimal usage of energy consumption by reduction of routing overhead. This method has been proposed to enhance the throughput and network lifetime. Our main contributions in this work are as follows.

1) This work propose the network topology, communication protocol and frame format for effective routing of aggregated data 2) In this paper the computational complexity of proposed algorithm is discussed 3) We build up a simulation setup that demonstrates the performance analysis of proposed method near to the real environment.

The proposed automated utility network topology mainly consist of sensors, integrated smart meter, cluster heads, base station and utility management servers [6].

Three type of sensors used to sense the flow of electricity, water, and pipelined gas. These sensors are connected to processing node called smart meter which consists of transmitter and receiver. The cluster heads carry forward data to BS in the network. The base station is located in central place, which receives data from various nodes in the network. The data is stored and processed in the servers. The architecture of proposed integrated public utility is shown in Figure 1. The details of each element are discussed in following sections.

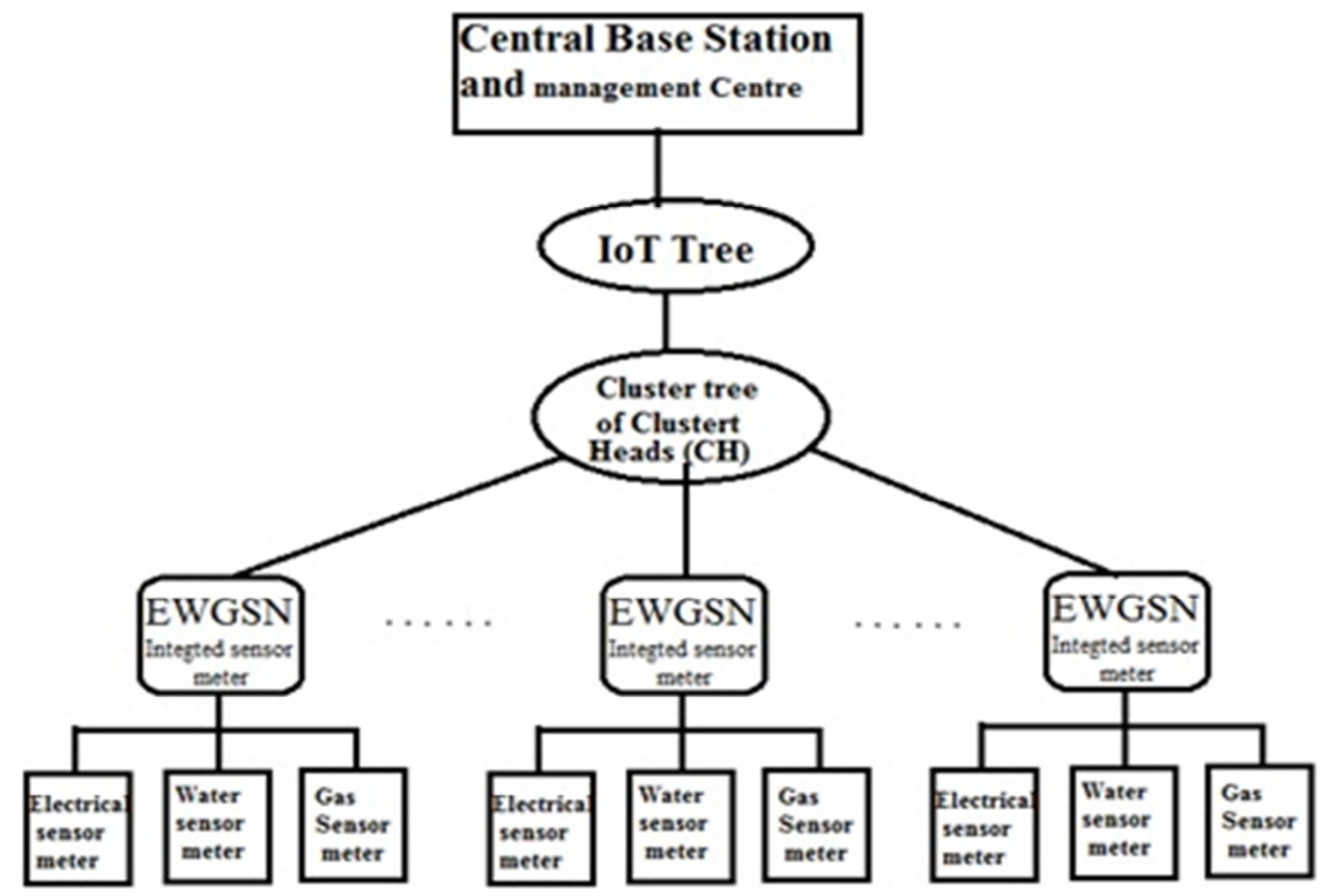

Figure 1. Architecture of integrated public utility services using WSN and IoT. 


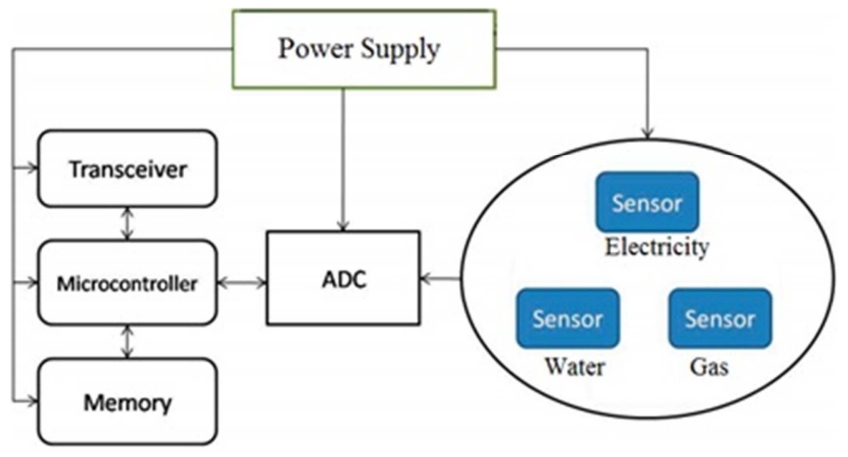

Figure 2. Architecture of Integrated Metered Sensor Meter.
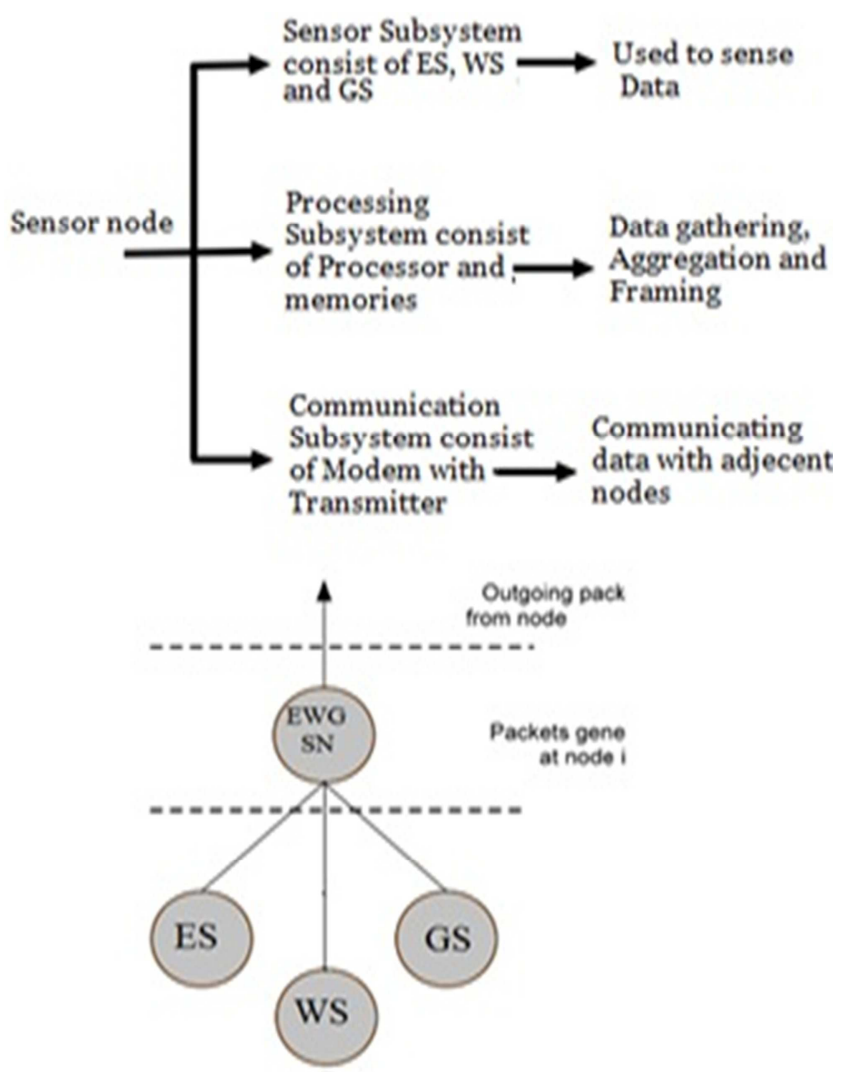

Figure 3. Data Processing Architecture of Integrated Metered Solution.

Integrated Smart Meter: In the proposed automated utility service network, We integrate electrical, water and gas metered utility services into single smart meter called EWGSN as shown in figure 2. In a smart city environment, each house consists of electrical, water and gas flow sensors. These flow sensors are used to measure utility usage. These flow sensors are integrated into single node named as EWGSN (i.e. Electrical, Water and Gas Sensor Node) as shown in figure 3 . The utility sensors used in EWGSN are flow sensors of electrical, water and gas. The flow sensors sense the flow of water, gas, and electricity. In this architecture, the EWGSN does preliminary functions such as collection of data from three utility sensors and store into the single node with RFD functionality [8]. Hence, EWGSN node is considered as level one data aggregation and fusion center as shown in Figures 2 and 3. Secondly, it packetizes sensors flow metered data into the standard frame as shown in Table 1 is basic frame structure and transmits to next $\mathrm{CH}$. This $\mathrm{CH}$ aggregates data of its members.

ES-Electrical flow Sensor, WS-water flow Sensor, GS-gas flow Sensor, EWGSN-Electrical, water and gas Sensor Node.

\section{Data Aggregation and Routing Scheme for Automated Public Utility Services Using WSN}

The proposed routing scheme is achieved in three phases [7]. First phase is setup phase; second phase is data gathering and aggregation phase. In the third phase is data routing. The routing phases are explained in the following section.

Setup Phase: Consider a homogeneous public utility network of metered 'n' sensor nodes (i.e EWGSN) and a BS distributed over a given locality. The location of the EWGSN and the BS are set and known prior to routing. We divide the entire residential area of the smart city in zones. These zones have a uniform number of lanes and integrated smart meters installed at every house as shown in Figure 1. In given utility WSN, few EWGSN become $\mathrm{CH}$ to collect all traffic from their respective member cluster. Once $\mathrm{CH}$ is selected, based on energy and distance, member nodes add themselves to the $\mathrm{CH}$. During the data transmission phase, the $\mathrm{CH}$ structures a TDMA-based schedule for each connected member to communicate with the $\mathrm{CH}$. The $\mathrm{CH}$ aggregates the data and then sends it to its central $\mathrm{BS}$. The $\mathrm{CH}$ is responsible for assigning gateways to the neighbouring clusters. The $\mathrm{CH}$ learns information about network connectivity from link updates. Each $\mathrm{CH}$ in the network learns the information about its neighbors from received Hello packets, and stores this information in its neighbor routing table. Periodically, it sends the update about its incoming links to its $\mathrm{CH}$. The $\mathrm{CH}$ in the network dynamically change based on the available energy in it and link faults during the lifetime in order to distribute the extra workload and energy consumption.

Table 1. Basic Frame from Node with example value.

\begin{tabular}{llll}
\hline Main Field & Example Value & Sub Field & Size \\
\hline Sync & 0x55 & & $8 \mathrm{~b}$ \\
DMAC & 0x6DE & & $12 \mathrm{~b}$ \\
SMAC & 0X9F2 & $12 \mathrm{~b}$ \\
C/D & 0X02 & $2 \mathrm{~b}$ \\
No of Nodes & $0 \times 01$ & & $8 \mathrm{~b}$ \\
Node Status Flag & 0X01 & & $2 \mathrm{nb}$ \\
Data Aggregate Flag & 0X7 & $3 \mathrm{~b}$ \\
Time Stamp & 0X7243C & Electrical & $14 \mathrm{~b}$ \\
SN Data & 0X1C2 & Water & $12 \mathrm{~b}$ \\
& 0X7AD & Gas & $12 \mathrm{~b}$ \\
& 0X5A4 & & $16 \mathrm{~b}$ \\
\hline
\end{tabular}

Table 2. Aggregated Data frame Cluster Head.

\begin{tabular}{llll}
\hline Main Field & Example Value & Sub Field & Size \\
\hline Sync & 0x55 & $8 \mathrm{~b}$ \\
DMAC & 0x8E3 & $12 \mathrm{~b}$ \\
\hline
\end{tabular}




\begin{tabular}{llll}
\hline Main Field & Example Value & Sub Field & Size \\
\hline SMAC & 0X9D5 & & $12 \mathrm{~b}$ \\
C/D & 0X1 & & $2 \mathrm{~b}$ \\
No of Nodes & 0X03 & & $8 \mathrm{~b}$ \\
Node Status Flag & 0X3 & $2 \mathrm{nb}$ \\
Data Aggregate Flag & $0 \times 02$ & & $3 \mathrm{~b}$ \\
Time Stamp & 0X3864 & Electrical & $14 \mathrm{~b}$ \\
SN1 Data & 0X213 & Water & $12 \mathrm{~b}$ \\
& 0xC33 & Gas & $12 \mathrm{~b}$ \\
& 0X0 & & $3 \mathrm{~b}$ \\
Data Aggregate Flag & 0X3 & & $14 \mathrm{~b}$ \\
Time Stamp & 0X17E3 & Water & $12 \mathrm{~b}$ \\
SN2 Data & 0X8A31 & & $3 \mathrm{~b}$ \\
Data Aggregate Flag & 0X2 & & $14 \mathrm{~b}$ \\
Time Stamp & 0X6211 & Electrical & $12 \mathrm{~b}$ \\
& 0X65ED & Water & $12 \mathrm{~b}$ \\
SN3 Data & 0X0 & Gas & $12 \mathrm{~b}$ \\
& 0x5CD2 & & $16 \mathrm{~b}$ \\
CRC 16 & 0XC33E & &
\end{tabular}

Data gathering and Aggregation Phase: Data aggregation technique uses a collection of data of member EWGSN nodes together and preventing transmission of redundant, non-usable routing information in packets over the network [4], [10]. This method is effective in reducing the number of packets to be sent over the public utility network. This characteristic decreases the transmission of routing overheads when previous $\mathrm{CH}$ give aggregated data to new $\mathrm{CH}$ in the next hop as shown below. In the proposed routing scheme and frame format, the fields such as active members, node flag, and data aggregation flag are used to identify redundant and repeated routing data from the member nodes and inform same to next hop to avoid searching unusable data. Every $\mathrm{CH}$ acts as data aggregation. Periodically each $\mathrm{CH}$ updates its members data connected to BS using control frame by enabling $\mathrm{C} / \mathrm{D}$ bit in the frame. $\mathrm{CH}$ updates periodically member's data such as a number of nodes, active and dead nodes/sensors and MAC IDs of each node to BS. BS keeps track of all member nodes in the cluster. BS collects the data through $\mathrm{CH}$ and updates member data as per $\mathrm{CH}$ member record byte. All the EWGSN sensor nodes and $\mathrm{CH}$ in the public utility network are immobile. The fixed BS is located far from all basic sensor node. Since TDMA is used to collect data from sensor nodes, different time interval and equal energy level from source to destination are considered during transmission and reception at each $\mathrm{SN}$. Let $\mathrm{K}_{\mathrm{n}}$ be the length of the message in bits, the parameter ' $L$ ' is the distance between the source node and receiver node. Radio propagation model will decide the distance thresh-old value in the network. For our analysis it is consider five sensor nodes SN1, SN2, SN3, SN4, SN5 and five cluster heads C1, $\mathrm{C} 2, \mathrm{C} 3, \mathrm{C} 4, \mathrm{C} 5$. This can be represented as $\mathrm{C} 1=[(\mathrm{SN} 1, \mathrm{Ts} 1)$, $(\mathrm{SN} 5, \mathrm{Ts} 2) \ldots . ..], \mathrm{C} 2=[(\mathrm{C} 1, \mathrm{Ts} 1),(\mathrm{SN} 2, \mathrm{Ts} 2) \ldots . . .],. \mathrm{C} 3=[(\mathrm{C} 2$, Ts1), (SN3, Ts2)......], C4=[(SN4, Ts1).............. $\mathrm{C} 5=[(\mathrm{C} 3, \mathrm{Ts} 1),(\mathrm{C} 4, \mathrm{Ts} 2) \ldots . . .$.

Where $\mathrm{CH}_{\mathrm{n}}$ is cluster head, $\mathrm{SN}_{\mathrm{n}}$ is sensor node and $\mathrm{T}_{\mathrm{s}}$ is time slot allotted by $\mathrm{CH}_{n}$ at given location.

In the proposed smart utility network is based on cluster tree topology between sensor meters as shown in Figure 1. In a WSN, the clustering scheme provides better services that can be leveraged by various wireless applications to achieve scalability. For example, it can be used to scale a service location and discovery mechanism in WSN. In integrated public utility sensor networks, flow sensors in EWGSN and $\mathrm{CH}$ might generate significant redundant data. Similar data in the packets from multiple nodes can be aggregated to reduce the number of transmissions during routing. The basic frame structure and aggregated frame carried by member node and $\mathrm{CH}$ data respectively is shown in the frame format with example values are shown in table 1. A frame carrying integrated metered aggregated data by cluster head is shown in Table 2 with example values. In the WSN based utility networks, data routing and transmission is the most power consuming activity of a sensor node. Data transmission must be kept to an absolute minimum to reduce overall power consumption of network. This can be achieved by reducing the amount of network traffic while routing data to BS. In order to reduce the amount of traffic in the public utility service network using WSN during routing, we propose novel data frame format and routing algorithm which carries integrated sensor data reading of metered public utility services data. The data is processed, aggregated and routed as per algorithm was given in the algorithm pseudo code 1 and 2. The data frame from $\mathrm{CH}$ carrying aggregated to next hop as shown in Figure 2. The proposed frame format is fault tolerant and supports scalability for the possibility to enlarge and reduce the network. The benefit of proposed integrated utility data frame is that it carries integrated metered flow sensor data in the single frame along with special control requests from $\mathrm{CH}$ for data gathering and aggregation.

Data Routing Phase: Once CH's are selected to form cluster tree. All the CH's in the network will send hello messages to BS through cluster tree until they get acknowledgment from BS which also consist of hop count. This information is helpful to determine the number of hops between $\mathrm{BS}$ and native $\mathrm{CH}$. Based on this the native information of $\mathrm{CH}$ are exchanged with nearest $\mathrm{CH}$ 's. The routing information exchange uses control packet during network setup time. To determine the best routes between the sensor node and BS in the multi-hop, $\mathrm{CH}$ use Belman ford, algorithm. Each $\mathrm{CH}$ stores number of member connected and members MAC ID, number of hops to BS and information about nearest best CH's. The best nearest $\mathrm{CH}$ information will help native $\mathrm{CH}$ for building a fault-tolerant network. Each $\mathrm{CH}$ can store routing information in the form of the table. The filling of tables occurs using query based communication. Since number hops degrade the performance of network due to data traffic delay in the router. Our proposed real-time system architecture is composed of IoT gateways to the utility servers and smart sensor meters. A utility such as electricity, water, and gas consumption data by the EWGSN at each home is forwarded to a central integrated utility management server system. The consumption data is forwarded to next $\mathrm{CH}$ towards BS. The redundant data of members such as Sync, DMAC, SMAC and $C / D$ is removed while routing aggregated data to next hop. When $\mathrm{CH}$ receives data from member nodes, it removes 
the redundant overhead and integrate the useful data to aggregates the utility information into one single packet. This integrated data packet is transmitted to the BS through many hops in tree topology. In our proposed method, during routing, this process is continued until the final metered sensor data is received by the BS. This method of data aggregation during routing is one of the most significant techniques which can be used to achieve traffic optimization in routing operation. This saves transmission energy efficiently and enhances the network lifetime and throughput of network. The algorithms for routing with data aggregation and communication as shown in figure 4 and figure 5 for algorithm 4 and 5 respectively.

1) Data Routing Algorithm Pseudocode: In this section, we present pseudo code of data routing at various stages in the network. The Setup phase, Data Fusion and Data aggregation are shown in figure 4 pseudo code Algorithm-1. The Data routing algorithm is shown in figure 5 Algorithm-2.

Algorithm 1 Data Aggregation at CH Psuedocode. Input: Response from various EWGSN and meter reading data. Output: Aggregated data to next hop $\mathrm{CH}$.

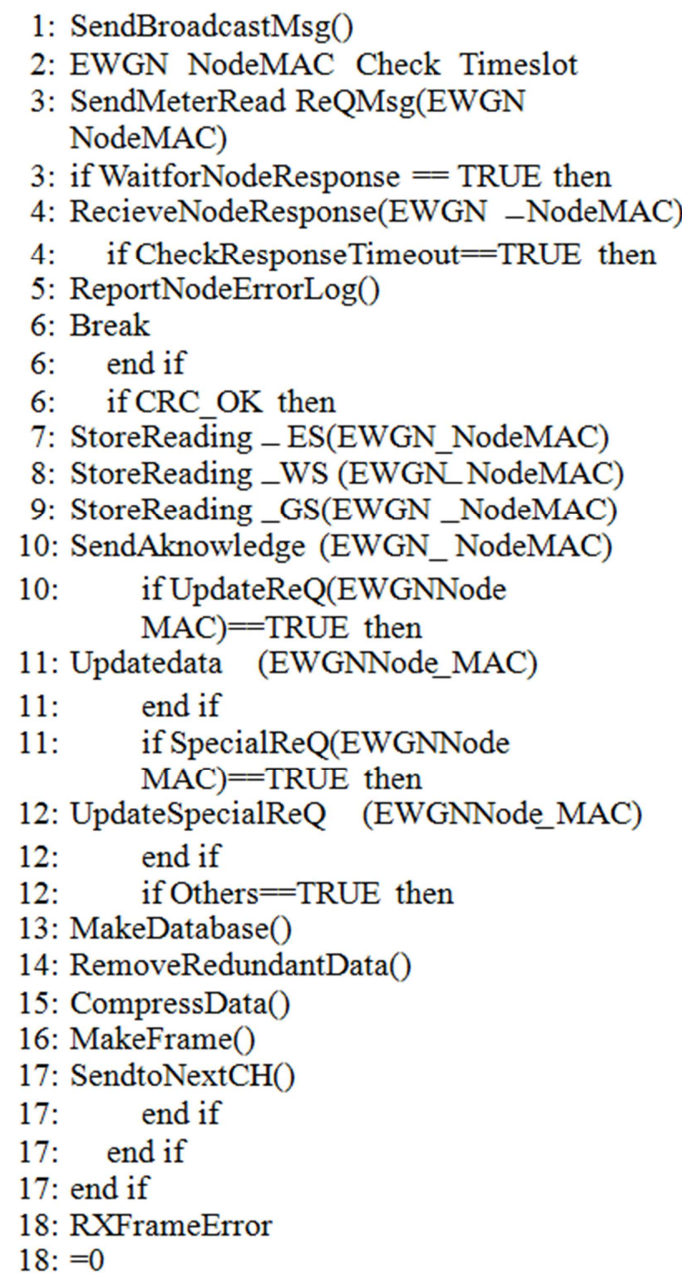

Figure 4. Data Aggregation at $\mathrm{CH}$.

Algorithm 2 Data Routing Algorithm Pseudo code. Input: Link status message. Output: Routing aggregated data.

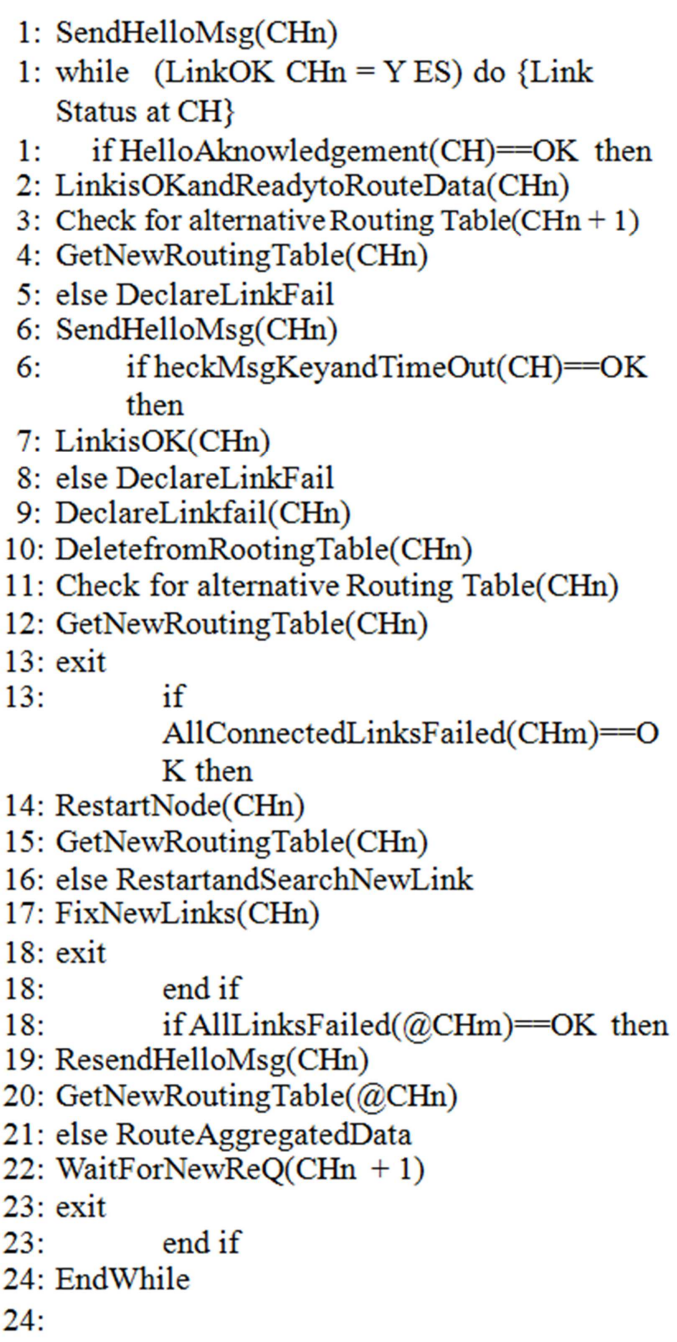

Figure 5. The Data routing algorithm.

\section{Computational Complexity of Proposed Data Routing With Data Aggregation Algorithm}

In this section we discuss computational complexity of proposed routing protocol with data aggregation for time, message and energy complexities.

A.Computational Complexity of Proposed Data Aggregation Algorithm

The efficient data processing of the gathered information is a key functionality in WSN. In the proposed data aggregation, we compute the time complexity, message complexity, and energy cost complexity of data aggregation processing operations for a cluster based multi-hop WSN of ' $n$ ' nodes. Typically, an aggregate (or summarized) value is computed at the data sink by applying the corresponding aggregate function such as MAX, COUNT, AVERAGE or MEDIAN to the collected data.

Let $\mathrm{M}, \mathrm{T}$, and $\mathrm{E}$ be the approximation ratio of an data aggregation algorithm in terms of message complexity, time complexity, and energy complexity respectively. To focus on the complexities of various data operations in the pro-posed 
utility WSN. Thus, it is assumed a simple model. It is assumed that there are $n+1$ metered sensor nodes $S_{n}=S_{n 0}$, $\mathrm{S}_{\mathrm{n} 1}, \mathrm{~S}_{\mathrm{n} 2}, \ldots . ., \mathrm{S}_{\mathrm{n}}$ that are deployed in a predefined region, where $S_{n 0}$ is the sink node. Each $S_{n}$ corresponds to a vertex in a graph $G$. The two vertices are connected if their corresponding $S_{n}$ can communicate directly. Then graph $G$ is called the communication graph of this utility network. when a node $S_{n i}$ sends data to a neighbouring node $S_{n j}$ on reliable link, then total message cost is only 1 .

Each metered node has an ability to monitor the utility usage and collect fixed data. It is assume that $X=d_{1}, d_{2}, \ldots$, $\mathrm{d}_{\mathrm{N}}$ is a total data of $\mathrm{M}$ elements collected by all $\mathrm{S}_{\mathrm{n}}$ nodes. The $M$ is the cardinality of set $X$. Each node $S_{i}$ has $d_{i}$ amount of raw data that can be denoted as $\mathrm{X}_{\mathrm{i}} \subset \mathrm{X}$. T hen $\mathrm{X}_{1}, \mathrm{X}_{2}, \mathrm{X}_{\mathrm{ni}}$ is called a distribution of $A$ at sites of $S_{n}$. We assume that one packet can contain multiple data as per proposed packet format (i.e., the packet size is at the order of $P(\log n+\log U)$, where ' $n$ ' is number of nodes and $U$ is the upper bound on values of $d_{i}$. The o node respond to BS based on a TDMA schedule and each packet is transmitted in one time slot. To optimize energy consumption, we assume that the minimum energy consumption by a node $\mathrm{S}_{\mathrm{n} 1}$ to send data correctly to a

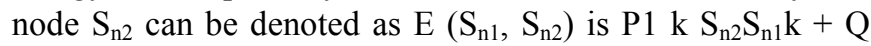
$\left(\mathrm{S}_{\mathrm{n} 2}\right)$, where $\mathrm{P} 1$ and $\alpha 2$ are constants depending on the environment, and $\mathrm{Q}\left(\mathrm{S}_{\mathrm{n} 2}\right)$ is the receiving cost of the node $\mathrm{S}_{\mathrm{n} 2}$.

B. The Message Complexity And The Energy Complexity of Proposed Data Aggregation Scheme:

The we use worst case measures to evaluate the performance of a proposed scheme. The message complexity and the energy complexity of a protocol is defined as the maximum number of total messages and the total energy consumed respectively by all nodes in a given geographic area and all possible data distributions.

For given geographic are the distributive function $Z_{i}$ and $a$ data aggregation tree $T$. The message complexity clearly is the number of edges in $T$, which is fixed as $S_{n}$. The energy cost complexity clearly is the total energy-cost used by all ' $n$ ' links in multi hop. This can be easily found using minimum STA (Spanning Tree Algorithm) where the link cost of $\mathrm{L}_{n}$ is the energy-cost for the communication. The time complexity of data aggregation depends on the time schedule $\mathrm{T}_{\mathrm{s}}$. A time schedule $T_{S}$ is valid for data aggregation of $X$ using tree $T$, if for every node $S_{n}$ is scheduled to transmit data at a time slot $\mathrm{T}_{\mathrm{s}}$ as per TDMA based on data received from all of its metered nodes in the utility network. Consequently, the timecomplexity of any data aggregation scheme for a utility network $G$ is at least the height of the tree. In terms of the message complexity, there is a graph $\mathrm{G}$, such that $(\mathrm{n} \log \mathrm{h})$ messages are required to compute the $\mathrm{N}^{\text {th }}$ smallest element in $\mathrm{G}$ and with probability at least $1 / \mathrm{nK}$ for every constant $\mathrm{K}<$ $1 / 2$, where $\mathrm{h}=\min (\mathrm{K}, \mathrm{NK})$. This present proposed algorithm that achieves this bound with high probability.

The time complexity is defined as the elapsed time is the time when the first message was transmitted to the time when the last message was received. If communication bandwidth is small, the data aggregation time complexity problem depends on the size of the network and even when the diameter of the network is constant.

Definition: Let $L$ be shortest path from source $S_{i}$ to Sink $S_{n}$ in the graph $\mathrm{G}$.

The total number of transmission for the optimal data aggregation required in this case is:

$$
\mathrm{T}_{\mathrm{r}}=\mathrm{L} 1+\mathrm{L} 2+\mathrm{L} 3 \ldots . . \mathrm{Ln}=\operatorname{sum}\left(\mathrm{L}_{\mathrm{i}}\right)
$$

Let number of transmissions required for proposed data aggregation protocol is $\mathrm{T}_{\mathrm{d}}$. The 'diameter' $\mathrm{D}$ of set of nodes

graph $G$ is $D=$ MaxI, $j<=\operatorname{DP}(i, j)$. Where DP (i.j) is shortest number of hops need to go from node $S_{i}$ to node $S_{j}$ in G.

Theorem: if the diameter $\mathrm{D}<\min (\mathrm{L})$, then $\mathrm{T} \mathrm{d}<\mathrm{T}$. In other words proposed data aggregation protocol perform better than optimal protocol in-terms of total number of transmission. Proof: if the sources S1, S2, S3,...... Sn have diameter $\mathrm{D}>=1$, the total number of transmission $\mathrm{T}_{\mathrm{d}}$ required for optimal protocol satisfies the following bound.

$$
\begin{gathered}
\mathrm{T} \mathrm{d}<=(\mathrm{K}-1) \mathrm{D}+\min (\mathrm{L}) \\
\mathrm{T} \mathrm{d}>=\operatorname{Min}(\mathrm{L})+(\mathrm{K}-1)
\end{gathered}
$$

Assume D and $\mathrm{K}$ are constant.

The proposed algorithms for data aggregation have optimum constant factors for time complexity and message complexity. The minimum energy data aggregation can be done by removing redundant data. The algorithm for data aggregation can achieve approximation ratio $\mathrm{T}$ for time complexity and $\mathrm{E}$ for energy complexity with $\mathrm{T} E=(\Delta)$. In other words, our method achieves the best trade-offs among the time complexity, message complexity and energy complexity with

$$
\mathrm{T}=(1), \mathrm{M}=1, \mathrm{E}=(\Delta) .
$$

\section{Computational Complexity of Data Routing In Public} Utility Network

The basic principle of any router will have routing tables. In the network every node keeps a table with routing entries. Using these entries, router could be determined via which link a message had to be sent to next hop. Effective routing with routing tables is a dominant strategy. There are lots of methods to reduce the memory requirement for the data holding in router. The proposed aggregation is one of the most important. It is based on removal of redundant information as per proposed method for each node in network. This way one can excessively reduce the size of routing tables.

We assume a standard abstract Random Access Machine (RAM). We will assume a RAM with Logarithmic cost criterion (i.e number of bits) for analysis of space complexities and unit cost for time (number of operations) complexities. The automated utility network is modeled as simple graph $\mathrm{G}=(\mathrm{V}, \mathrm{E})$, where nodes from $\mathrm{V}$ represent processors and edges $\mathrm{E}$ are communication links. The maximum degree of $\mathrm{G}$ is denoted by.

The proposed routing algorithm contains a description of a 
routing algorithm in pseudo code. The routing table at $\mathrm{CH}$ is given in table. Input: destination node 'd', message ' $m$ ' etc as per table.

A data structure table given in $\mathrm{CH}$ table field which implements the routing table. Its size is $\mathrm{n}$ and it stores identification labels. It is organized in such a manner that fields stored an ID-number of port via which a message addressed to a node ' $w$ ' is forwarded.

Lemma 1. To store a positive integer ' $i$ ' it is required a register with at least $[\log 2 \mathrm{i}]$ bits.

Proof. It is possible to vary at most $i=2 n$ values in $n$-bit register. Taking logarithm of $i=2 n$ resulted in $\log 2 i=n$. It means for storing the ' $i$ ' value is an $n$ bit register necessary. Theorem 1. Routing with routing tables requires

$\log (\mathrm{n} \log \Delta)$ bits to store the routing information.

Proof. The size of registers in RAMs depends on the information fields stored in it as bits. Hence the memory requirements are $n .(\log 2 \Delta)=\log (n \log \Delta)$.

Theorem 2 . Time complexity of routing algorithm in RAM model with unit cost criterion is (1).

Proof. Routing Algorithm consists of 5 operations.

It is necessary to carry out exactly these (1) $=5$. Operations for constant amount of data input. For variable frame size based on aggregated data frame at $\mathrm{CH}$ the complexity of binary searching is $(\log n)$ for ' $n$ ' fields. Assuming the routing algorithm the resulting complexity is $(\log (\mathrm{Y})=(\log (\mathrm{k} \Delta))$.

\section{Comparative Performance Analysis of Proposed Data Routing With Data Aggregation Methodology}

In this section the comparative performance analysis of the proposed method with previous similar methods are evaluated by simulation in QUALNET environment.

For the comparative analysis of our two main objectives data aggregation and routing of utility data over WSN networks, we have considered two previous approaches a) ECWR and b) EHWMP used for meter data routing for integrated utility management. They are disused as follows.

We use QUALNET Simulator with customized C++ functions to compare our results with two previous approaches a) ECWR and b) EHWMP. This was done primarily because the simulator does not have any built in support for correlated events and data of previous research works.

By analyzing the work flow of ECWR and E-HWMP protocols, it can be found that the $\mathrm{CH}$ with data aggregation is not considered in routing selection. So it is necessary to adjust the original parameters of previous works for comparative analysis in the simulation environment. Nodes and $\mathrm{CH}$ with data aggregation capability should be considered when the data is transmitted in the next hop $\mathrm{CH}$. The simulation adjustment includes making common simulation environment as shown in table, modifying the area, number of transmission, and specification of nodes.
In this research, an attempt is made to study and compare the performance of proposed IPUS data routing along with data aggregation (IPUS_DAR) with presently available two previous approaches a) ECWR and b) EHWMP.

Table 3. Simulation Parameter Setup Data Routing.

\begin{tabular}{ll}
\hline Parameter. & Parameter value. \\
\hline Standard. & WSN. \\
Number of nodes. & 10 to 100. \\
Area. & 500 x 500 Meters. \\
Simulation time. & 2 Mins. \\
Frequency. & $2.4 \mathrm{GHz}$. \\
PHY and MAC. & 802.15 .4$. \\
Modulation. & OQPSK. \\
TX Power. & 0 dBm. \\
Energy Model. & MICAZ mote. \\
Path loss Model. & Two ray Model. \\
Packet Rates. & $10 \mathrm{kbps}$ \\
Battery Model. & $1200 \mathrm{mAhr}$ Linear Model. \\
Antenna. & Omni-directional. \\
Deployment. & Fixed. \\
\hline
\end{tabular}

The performance metrics Packet Delivery Ratio (PDR), Throughput and End to End Delay are evaluated for multihop with varying number of nodes and number of hops in known size of area.

\section{A. Simulation Environment}

For comparative analysis of our work with previous approaches a) ECWR and b) EHWMP, we use the QUALNET simulator for our simulations. The network consists of 100 nodes for predefined confined in a $500 * 500$ $\mathrm{m}$ area. Trans-mission range of each node is assumed $20 \mathrm{~m}$. The simulation runs for 2 minutes. BS is located on the outermost area.

We simulate three CBR flows originating from randomly chosen nodes across the network. Each flow sends 32 byte packets at $10 \mathrm{kbps}$. We generate and evaluate different possible random and predefined scenarios for simulation. The key performance measures are energy consumption and routing overhead. The results presented here are the average values taken from multiple simulation results.

B. Results

We generate and evaluate possible predefined scenarios for simulation. The results presented here are the average values taken from multiple simulation results. The number of nodes as is incrementally varied as, 10, 20, 30 till 100 and the above performance metrics are evaluated. Clusters are formed such that the average size of each cluster is 10 Percentage of the total number of nodes.

The figure 6 EED vs Number of nodes shows the end to end delay occurred for three methods, when the number of nodes is increased. it is observable that the number of nodes increases, the delay increases linearly, as the inter-cluster path length will be high. However, IPUS DAR chose shortest paths between each $\mathrm{CH}$ for inter-cluster routing along with data aggregation, the delay is $10 \mathrm{sec}$ less when compared to ECRM and $15 \mathrm{Sec}$ less when compared to EHWMP. 


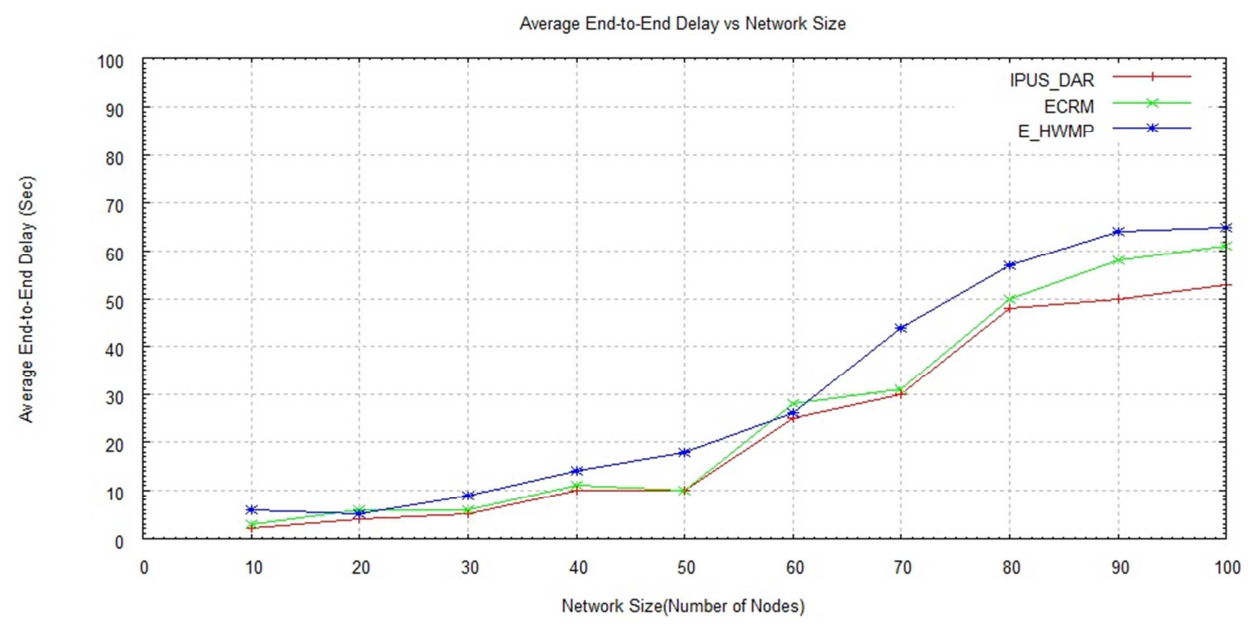

Figure 6. Average End-to-End to Delay vs Network Size.

Figure 7 PDR vs Number of nodes show the packet delivery ratio of three methods when the number of nodes is increased. Their will be more packet drops in multi-hop transmission. This is due to the fact that, when there are more nodes, the load of the $\mathrm{CH}$ increases leading to more packet drops in case of ECRM and E-HWMP. Due to data aggregation at each $\mathrm{CH}$ in hops during inter-cluster routing, the packet delivery ratio is 9 percentage high, when compared to ECRM and 20 percentage high when compared to EHWMP.

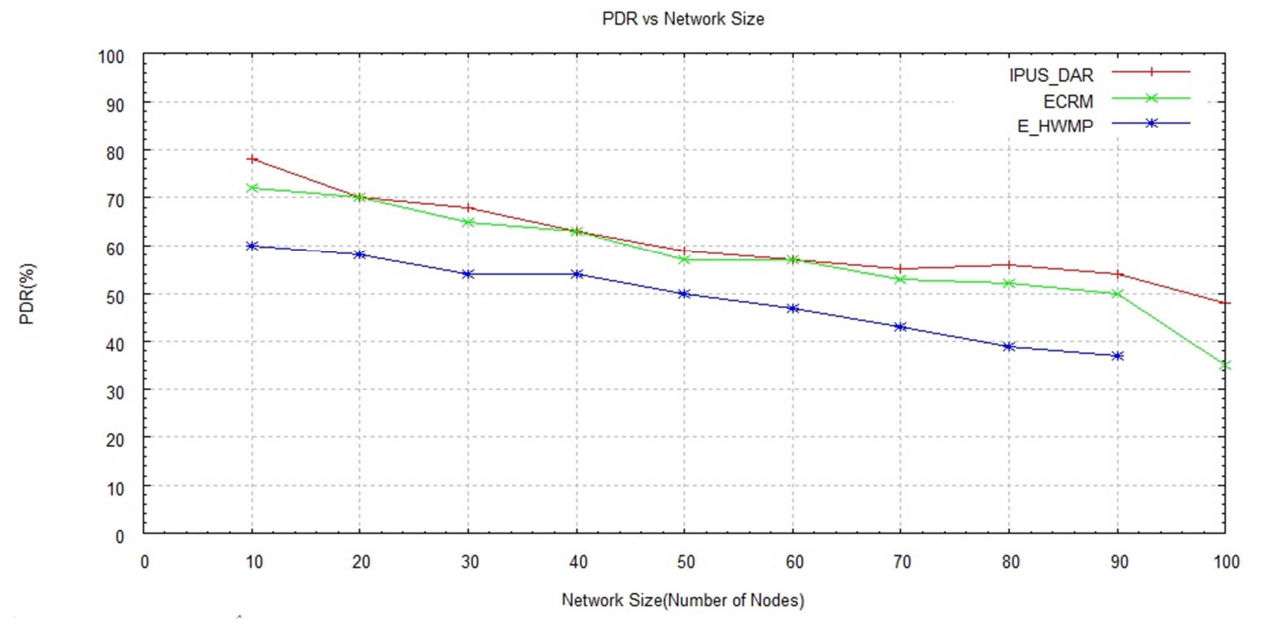

Figure 7. PDR vs Network Size.

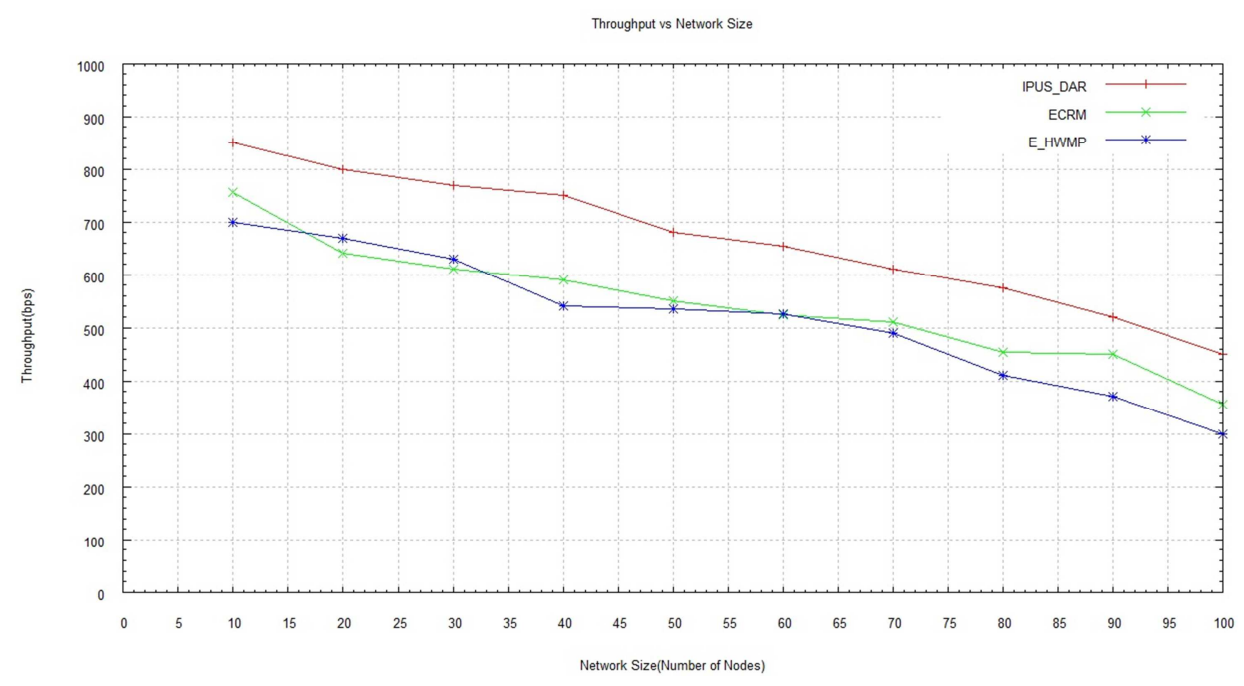

Figure 8. Throughput vs Network Size. 
Figure 8 Throughput vs Number of nodes shows the throughput with varying number of nodes for three methods, when the number of nodes is increased. As it can be seen that as the number of nodes increases, the amount of data in the network increases linearly in multi hop cluster network. However, IPUS DAR chose data aggregation at each $\mathrm{CH}$ for inter routing, the throughput is 8bps high when compared to ECRM and is 15 bps high when compared to EHWMP.

\section{Conclusion}

In this work, we present architecture for integrated utility metering service that include electricity, water and gas AMR and methodology for data aggregation and routing for integrated public utility services using WSN. This work aims to minimize redundant routing data in the network by aggregating data and improve traffic routing performance. The proposed IPUS-DAR is a new routing protocol that focuses on the reduction of routing overhead and energy consumption. This increase throughput and network lifetime. As the main contribution of this work, we highlight the creation of frame format, protocol and algorithms for data routing of integrated metered data such as electricity, water, and gas. We believe that this method improve the data routing performance in automated smart meter management of public utility services network. Finally, we present complexity computations of novel data aggregation and routing algorithms and performance analysis of proposed method with previous works.

The future work should includes thorough analysis in more scenarios with varying network size based numbers of sensor nodes. Evaluating alternative algorithms for $\mathrm{CH}$ selection during changes in topology due to addition or deletion of nodes or fault in network. The long term research in this area should focus on development of the new routing algorithms for efficient data aggregation during data transmission in smart public utility network. Our approach should give solutions for challenges in topology construction, data routing and loss tolerance smart utility network by including several optimization techniques. In addition to implementing these techniques, further research should concentrate on reducing message costs and improve tolerance to leakage, theft and failure in automated utility network. We intend a future work, to evaluate the proposed routing methodology in several smart public utility grid scenarios, making it as general as possible and transforming it in a methodology for efficient planning and deployment of automated public utility data for smart city implementation using WSN.

\section{Acknowledgements}

We thank management and staff of REVA University for providing infrastructure to carry research in WSN based application and access to QUALNET simulator.

\section{References}

[1] A. El-Mougy A. Sabbah and M. Ibnkahla. Survey of networking challenges and routing protocols in smart grids. In IEEE Transactions on Industrial Informatics, (Vol. 10, Issue. 1), February 2014.

[2] M. Conti E. Ancillotti, R. Bruno. Rpl routing protocol in advanced metering infrastructures: An analysis of the unreliability problems. In Sustainable Internet and ICT for Sustainability (SustainIT), pages 1-10, October 2012.

[3] Yasir Faheem and Rehmat Ullah Byung Seo Kim. Energy and congestion aware routing metric for smart grid ami networks in smart city. In IEEE Coneference, pages 1-11, 2017.

[4] Wei Yuan. Srikanth V. Krishnamurthy and Satish K. Tripathi. Synchro-nization of multiple levels of data fusion in wireless sensor networks. In Proceedings of GLOBECOM, pages 221225, 2003.

[5] Xiaoguang HU Li LI. The architecture of wifi-based wsn for amr system and e-hwmp routing protocol. In Sensors and Transducers, Vol. 164. Issue 2, pages 80-91, February 2014.

[6] Rajashekhar C. Biradar Raja Jitendra Nayaka. Cluster based data aggregation in wireless sensor based network for public utility control and management. In International IEEE Conference on Advances in Electronics, Computers and Communications (ICAECC), pages 1-5, 2014.

[7] Rajashekhar C. Biradar Raja Jitendra Nayaka. Qos analysis of wsn based cluster tree data fusion for integrated public utility management. In International IEEE Conference on Advance Computing Conference (IACC), pages 579-584, 2015.

[8] Rajashekhar C. Biradar Raja Jitendra Nayaka. A survey on wireless net-work applications in automated public utilities control and management. In Journal of Telecommunications and Information Technology (JTIT), NIT, Warsa, Poland. Issue-3, pages 13-24, 2015.

[9] S. R. Das V. Kathuria, G. Mohanasundaram. A simulation study of routing protocols for smart meter networks. In IEEE International Conference on Smart Grid Communications (SmartGridComm), pages 384 - 389, October 2013.

[10] Y. S. Han W. Du. J. Deng and P. K. Varshney. A witnessbased approach for data fusion assurance in wireless sensor networks. In Proceedings of GLOBECOM, pages 1-5, 2003.

[11] ZhiliZhou. Optimal relay node placement in wireless sensor network for smart buildings metering and control. In IEEE International Conference Communication Technology (ICCT), pages 456-461, 2013.

[12] Zhaoli Zhang Liting Cao, Wei Jiang. Automatic meter reading system based on wireless mesh networks and sopc technology. In Intelligent Networks and Intelligent Systems (ICINIS), pages 142-145, 2009.

[13] Mohamed Afifi, Mohamed F. Abdelkader, Atef Ghoneim. An IoT System for Continuous Monitoring and Burst Detection in Intermittent Water Distribution Networks. International Conference on Innovative Trends in Computer Engineering (ITCE 2018), Aswan University, Egypt, pages. 240-247, 2018. 
[14] Thobekile Ngcobo, Farzad Ghayoor, Study the Topology Effect on a G3-PLC based AMI Network, 2019 SAUPEC/RobMech/PRASA Conference Bloemfontein, South Africa, January 28-30, pages. 629-633.2019.

[15] Lucas Novelli, Luisa Jorge, Paulo Melo, André Koscianski. Application Protocols and Wireless Communication for IoT: A Simulation Case Study Proposal. 11 ${ }^{\text {th }}$ International Symposium on Communication Systems, Networks and Digital Signal processing (CSNDSP), pages 1-6, 2018.

[16] Mustafa Burunkaya, Tufan Pars. A Smart Meter Design and Implementation Using ZigBee Based Wireless Sensor Network in Smart Grid. 4th International Conference on Electrical and Electronics Engineering (ICEEE), pages 158$162,2017$.

[17] Bibek Kanti, Shiv Nath Yadav, Shivam Kumar, Sadhan Gope. IOT Based Smart Energy Meter for Efficient Energy Utilization in Smart Grid. International conference on Power,
Energy and Environment: Towards Smart Technology (ICEPE). Pages 1-5. 2018.

[18] Almhana, Vartan Choulakian, Jalal Almhana, Catherine. An Efficient Approach for Data Transmission in PowerConstrained Wireless Sensor Network. IEEE International Conefrence on Communication (ICC) Ad-Hoc and Sensor Networking Symposium. Pages 1-6.2017.

[19] H. Ali, W. Y. Chew, F. Khan, Steven R. Weller. Design and Implementation of an IoT Assisted Real-Time ZigBee Mesh WSN Based AMR System for Deployment in Smart Cities. The 5th IEEE International Conference on Smart Energy Grid Engineering pages 264-270. 2017.

[20] Purusharth Semwal, Sourish Palit, Shlok Indulkar and S. Senthilmurugan. Smart Metering in Smart Grid. International Journal of Engineering and Advanced Technology (IJEAT) ISSN: 2249 - 8958, Volume-8 Issue-4, pages 1020-1027, April 2019. 